\begin{tabular}{|c|l|}
\hline Title & Non-self-similar, partial, and robust collapse of four point vortices on a sphere \\
\hline Author(s) & Sakajo, Takashi \\
\hline Citation & $\begin{array}{l}\text { Physical Review E, 78(1), 016312-016320 } \\
\text { https://doi.org/10.1103/PhysRevE.78.016312 }\end{array}$ \\
\hline Issue Date & 2008-07-22 \\
\hline Doc URL & http://hdl.handle.net/2115/34772 \\
\hline Rights & ○ 2008 A merican Physical Society \\
\hline Type & article \\
\hline File Information & GetPDFServlet.pdf \\
\hline
\end{tabular}

Instructions for use 


\title{
Non-self-similar, partial, and robust collapse of four point vortices on a sphere
}

\author{
Takashi Sakajo* \\ Department of Mathematics, Hokkaido University, Sapporo Hokkaido 060-8610, Japan \\ Presto, Japan Science and Technology Agency, Tokyo, Japan
}

(Received 30 March 2008; published 22 July 2008)

\begin{abstract}
This paper gives numerical examples showing that non-self-similar collapse can occur in the motion of four point vortices on a sphere. It is found when the four-vortex problem is integrable, in which the moment of vorticity vector is zero. The non-self-similar collapse has significant properties. It is partial in the sense that three of the four point vortices collapse to one point in finite time and the other one moves to the antipodal position to the collapse point. Moreover, it is robust with respect to perturbation of the initial configuration as long as the system remains integrable.
\end{abstract}

DOI: 10.1103/PhysRevE.78.016312

PACS number(s): 47.32.C-

\section{INTRODUCTION}

Interaction between coherent vortex structures plays a significant role in the understanding of inviscid and incompressible flows in two-dimensional Euclidean space as well as on the surface of a sphere, since vorticity is an invariant quantity along the path of a fluid particle. As a simple mathematical treatment to understand the interaction, we assume that the vorticity is concentrated in Dirac's $\delta$ functions, which are called point vortices. The motion of $N$-point vortices is often referred to as the $N$-vortex problem and it has been investigated as a model for two-dimensional Euler flows for more than a century. Owing to its mathematical simplicity, many research papers are now available. For a complete list of references on this subject, see the book by Newton [1].

We focus here on the case in which the number of point vortices is small. Since the motion of the point vortices is formulated as a Hamiltonian dynamical system with $N$ degrees of freedom [1], research results are stated in the framework of the Hamiltonian dynamical systems theory. Regarding the integrability, the three-vortex problems in the plane and on the sphere are both integrable for any strengths of point vortices [2-4]. Although the four-vortex problem is not integrable in general (e.g., [5]), it becomes integrable with a certain additional constraint condition. Such integrable fourvortex problems have been studied for the planar case $[7,8]$ and for the spherical case [6]. For $N \geqslant 5$, the $N$-vortex problem is no longer integrable in the plane as well as on the sphere.

On the other hand, it is known that under some circumstances, during their motion, point vortices can collapse to a single point. The existence of such a singular solution is physically important since the point-vortex model fails to approximate the Euler flow after the critical time. In addition, the singular solution is of mathematical significance, since its properties provide us with useful information that would contribute to theoretical research of the Euler flows. So far, only self-similar collapse, in which all point vortices shrink to a point self-similarly in finite time without chang-

\footnotetext{
*URL: http://www.math.sci.hokudai.ac.jp/ sakajo; sakajo@math.sci.hokudai.ac.jp
}

ing ratios between their relative distances, has been investigated. In the planar $N$-vortex problem, Kimura gave a necessary condition on the strengths of the point vortices for the self-similar collapse, and he also derived algebraic equations for the ratios [9]. By solving the algebraic equations, he constructed a self-similar collapse solution of the three point vortices in the plane. Another approach has been introduced to derive the self-similar collapse of the three-point vortices for the planar case [1] and for the spherical case [10]. However, it is still unknown whether non-self-similar collapse can occur or not. As for the planar three-vortex problem, it has been mathematically proven that non-self-similar collapse never occurs [11]. Hence, we need to consider at least the four-vortex problem in order to obtain non-self-similar collapse. Furthermore, we do not yet know whether some of the point vortices collapse to a point while the others remain away from the collapse point at the singular time.

In the present paper, we give numerical evidence that nonself-similar and partial collapse is possible in the integrable four-vortex problem on the sphere. In the next section, we review the results of the paper [6] on the motion of the integrable four point vortices on the sphere. Then, in Sec. III, we show an example of non-self-similar collapse on the sphere and describe its properties in detail. In Sec. IV, we give more examples to see what conditions are required for the existence of non-self-similar collapse. The last section contains a summary and discussion.

\section{INTEGRABLE MOTION OF FOUR POINT VORTICES ON SPHERE}

We summarize some results on the integrable four-vortex motion on the sphere given in [6], which helps us describe the collapse solution in the following sections. Regarding the detailed derivations of these results, we would like the readers to refer to the original paper.

Let $\left(\theta_{m}, \phi_{m}\right)$ and $\Gamma_{m}$ denote the spherical coordinates of the $m$ th point vortex on the unit sphere and its strength for $m=1, \ldots, 4$, respectively. With the Poisson bracket between two functions $f$ and $g$,

$$
\{f, g\}=\sum_{m=1}^{4} \frac{1}{\Gamma_{m}}\left(\frac{\partial f}{\partial \phi_{m}} \frac{\partial g}{\partial \cos \theta_{m}}-\frac{\partial g}{\partial \phi_{m}} \frac{\partial f}{\partial \cos \theta_{m}}\right),
$$

the motion of the point vortices is governed by 


$$
\frac{d \cos \theta_{m}}{d t}=\left\{\cos \theta_{m}, H\right\}, \quad \frac{d \phi_{m}}{d t}=\left\{\phi_{m}, H\right\},
$$

where the Hamiltonian is given by

$$
H=-\frac{1}{4 \pi} \sum_{j=1}^{4} \sum_{m>j}^{4} \Gamma_{m} \Gamma_{j} \log l_{m j}^{2}
$$

and

$$
l_{m j}^{2}=2\left[1-\cos \theta_{m} \cos \theta_{j}-\sin \theta_{m} \sin \theta_{j} \cos \left(\phi_{m}-\phi_{j}\right)\right]
$$

is the Euclidean distance between the $m$ th and $j$ th point vortices. Let us introduce the moment of vorticity vector $\boldsymbol{M}$ $=(Q, P, S)$ by

$$
\begin{gathered}
Q=\sum_{m=1}^{4} \Gamma_{m} \sin \theta_{m} \cos \phi_{m}, \\
P=\sum_{m=1}^{4} \Gamma_{m} \sin \theta_{m} \sin \phi_{m}, \\
S=\sum_{m=1}^{4} \Gamma_{m} \cos \theta_{m} .
\end{gathered}
$$

It follows from $\{H, Q\}=\{H, P\}=\{H, S\}=0$ that each component of $\boldsymbol{M}$ is invariant in time. Moreover, we have $\{Q, P\}$ $=S,\{P, S\}=Q$, and $\{S, Q\}=P$, and thus they are in involution with each other when $Q=P=S=0$ holds at the initial moment. This indicates that the four-vortex problem on the sphere is integrable if the moment of vorticity vector is zero [1].

In what follows, the motion of the four point vortices is described in terms of their relative distances $l_{m j}^{2}$. The integrable system admits two invariant quantities, the Hamiltonian $H$ and $C$,

$$
C=\Gamma^{2}=\sum_{j=1}^{4} \sum_{m>j}^{4} \Gamma_{m} \Gamma_{j} l_{m j}^{2},
$$

where $\Gamma$ denotes the total circulation, defined by $\Gamma=\Gamma_{1}+\Gamma_{2}$ $+\Gamma_{3}+\Gamma_{4}$. Due to the zero moment condition, the four-vortex problem can be reduced to a three-vortex problem with a method used by Rott in the planar four-vortex problem [12]. Namely, it follows from $Q=P=S=0$ that we have the relations between the distances $l_{m j}^{2}$ for $m, j=1, \ldots, 4$ as follows:

$$
\begin{gathered}
\Gamma_{1} \Gamma_{4} l_{14}^{2}=\Gamma_{2} \Gamma_{3} l_{23}^{2}+\Gamma\left(\Gamma_{1}-\Gamma_{2}-\Gamma_{3}+\Gamma_{4}\right), \\
\Gamma_{2} \Gamma_{4} l_{24}^{2}=\Gamma_{3} \Gamma_{1} l_{31}^{2}+\Gamma\left(-\Gamma_{1}+\Gamma_{2}-\Gamma_{3}+\Gamma_{4}\right), \\
\Gamma_{3} \Gamma_{4} l_{34}^{2}=\Gamma_{1} \Gamma_{2} l_{12}^{2}+\Gamma\left(-\Gamma_{1}-\Gamma_{2}+\Gamma_{3}+\Gamma_{4}\right) .
\end{gathered}
$$

Owing to these relations, the relative position of the fourth point vortex is determined by the configuration of the vortex triple 123.

In that paper [6], necessary conditions for self-similar collapse were also shown. It has been proven that there exist no self-similar four-vortex collapse and no self-similar binary

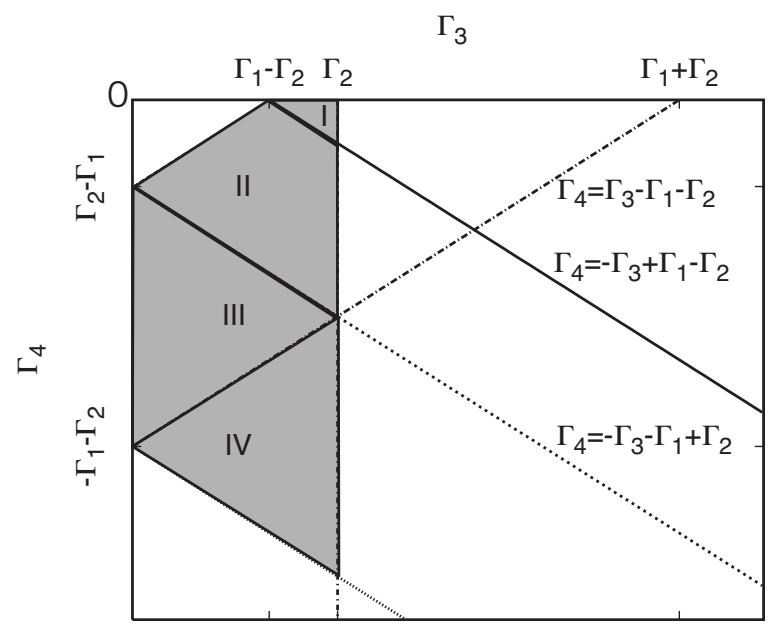

FIG. 1. Possible region (11) of $\left(\Gamma_{3}, \Gamma_{4}\right)$ for given $\Gamma_{1}$ and $\Gamma_{2}$.

collapse, in which pairs of two point vortices collapse at two different points simultaneously. However, the self-similar triple collapse, in which three of the four points collapse and the other one remains away from the collapse point, was not ruled out. For instance, the self-similar collapse of the vortex triple 234, which we deal with in this paper, is permitted when the vortex strengths satisfy the following conditions:

$$
\begin{gathered}
\Gamma_{1}-\Gamma_{2}-\Gamma_{3}-\Gamma_{4}=0, \\
\Gamma_{2} \Gamma_{3}+\Gamma_{2} \Gamma_{4}+\Gamma_{3} \Gamma_{4}=0 .
\end{gathered}
$$

The first condition comes from Eqs. (5)-(7) and the invariance of $C$ with the assumptions that $l_{23}^{2}=l_{34}^{2}=l_{24}^{2}=0$ and $l_{12}^{2}, l_{13}^{2}, l_{14}^{2} \neq 0$ at a certain time. The second one is derived from the invariance of $H$ with the additional self-similarity assumption, $l_{34}^{2}=\lambda_{1} l_{23}^{2}$ and $l_{24}^{2}=\lambda_{2} l_{23}^{2}$ for some constants $\lambda_{1}, \lambda_{2} \in \mathbb{R}$. These necessary conditions provide us with additional information on the triple collapse. Substituting Eq. (8) into Eqs. (5)-(7) leads us to

$$
l_{14}^{2}=\frac{\Gamma_{2} \Gamma_{3}}{\Gamma_{1} \Gamma_{4}} l_{23}^{2}+4, \quad l_{31}^{2}=\frac{\Gamma_{2} \Gamma_{4}}{\Gamma_{3} \Gamma_{1}} l_{24}^{2}+4, \quad l_{12}^{2}=\frac{\Gamma_{3} \Gamma_{4}}{\Gamma_{1} \Gamma_{2}} l_{34}^{2}+4,
$$

which indicates that if the vortex triple 234 collapses, i.e., $l_{23}^{2}=l_{24}^{2}=l_{34}^{2}=0$, then the first vortex is located at the antipodal position to the collapse point.

The strengths of the point vortices are further restricted due to the boundedness of the relative distances between point vortices on the sphere, i.e., $0 \leqslant l_{m j}^{2} \leqslant 4$. The existence region of $\Gamma_{3}$ and $\Gamma_{4}$ for given $\Gamma_{1}$ and $\Gamma_{2}$ is called the possible region. For instance, the triple 234 collapse is possible when $\Gamma_{1} \geqslant \Gamma_{2} \geqslant \Gamma_{3}>0>\Gamma_{4}$, for which the possible region is given as follows [6]:

$$
\Gamma_{1}<\Gamma_{2}+\Gamma_{3}-\Gamma_{4}, \quad-\Gamma_{4}<\Gamma_{1}+\Gamma_{2}+\Gamma_{3}, \quad \Gamma_{1}<2 \Gamma_{2} .
$$

Figure 1 shows the possible region (11), which is divided into four subregions, say $I$ to $I V$, by the lines $\Gamma_{4}=-\Gamma_{3}+\Gamma_{1}$ $-\Gamma_{2}, \Gamma_{4}=\Gamma_{3}-\Gamma_{1}-\Gamma_{2}$, and $\Gamma_{4}=-\Gamma_{3}-\Gamma_{1}+\Gamma_{2}$. Let us note that 


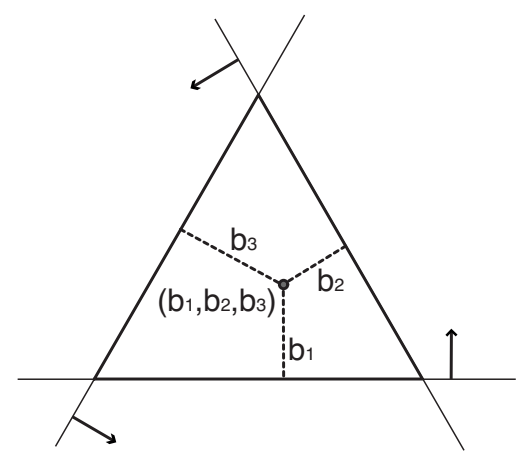

FIG. 2. Trilinear representation of the configuration of the vortex triple 123.

the first line is equivalent to the necessary condition (8) for which the triple 234 collapse is permitted.

The dynamics of the integrable system is represented in the trilinear coordinates, which is a standard and convenient tool for the description of integrable systems $[2-4,6,7]$. Now we suppose that $\Gamma\left(\Gamma-2 \Gamma_{4}\right) \neq 0$. Then the trilinear coordinates $\left(b_{1}, b_{2}, b_{3}\right)$ are defined by

$$
b_{1}=\frac{3 \Gamma_{2} \Gamma_{3} l_{23}^{2}}{\Gamma\left(\Gamma-2 \Gamma_{4}\right)}, \quad b_{2}=\frac{3 \Gamma_{3} \Gamma_{1} l_{31}^{2}}{\Gamma\left(\Gamma-2 \Gamma_{4}\right)}, \quad b_{3}=\frac{3 \Gamma_{1} \Gamma_{2} l_{12}^{2}}{\Gamma\left(\Gamma-2 \Gamma_{4}\right)} .
$$

Since $b_{1}+b_{2}+b_{3}=3$ due to Eqs. (5)-(7), the configuration of the vortex triple 123 corresponds to the point in the trilinear phase space, in which each component of the trilinear coordinates represents the distance from one of the sides of the equilateral triangle with height 3 as shown in Fig. 2. In addition, we use other trilinear coordinates $\left(B_{1}, B_{2}, B_{3}\right)$, in which

$$
\begin{aligned}
& B_{1}=\frac{3 \Gamma_{1} \Gamma_{4} l_{14}^{2}}{\Gamma\left(\Gamma-2 \Gamma_{4}\right)}=b_{1}+\frac{3\left(\Gamma-2 \Gamma_{2}-2 \Gamma_{3}\right)}{\Gamma-2 \Gamma_{4}}, \\
& B_{2}=\frac{3 \Gamma_{2} \Gamma_{4} l_{24}^{2}}{\Gamma\left(\Gamma-2 \Gamma_{4}\right)}=b_{2}+\frac{3\left(\Gamma-2 \Gamma_{3}-2 \Gamma_{1}\right)}{\Gamma-2 \Gamma_{4}}, \\
& B_{3}=\frac{3 \Gamma_{3} \Gamma_{4} l_{34}^{2}}{\Gamma\left(\Gamma-2 \Gamma_{4}\right)}=b_{3}+\frac{3\left(\Gamma-2 \Gamma_{1}-2 \Gamma_{2}\right)}{\Gamma-2 \Gamma_{4}} .
\end{aligned}
$$

The equalities on the right-hand sides are derived from Eqs. (5)-(7) and (12). Since we have $B_{1}+B_{2}+B_{3}=6 \Gamma_{4} /\left(\Gamma-2 \Gamma_{4}\right)$, each component of $\left(B_{1}, B_{2}, B_{3}\right)$ also represents the distance from one of the sides of another equilateral triangle with height $6 \Gamma_{4} /\left(\Gamma-2 \Gamma_{4}\right)$, which is called the physical triangle. Let us remark that the collapse of the vortex triple 234 corresponds to the point at the side of the trilinear triangle $b_{1}$ $=0$ and the vertex of the physical triangle $B_{2}=B_{3}=0$.

We need to take into consideration another constraint on the motion of the four point vortices. Since the vortex triple 123 must form a triangle on the sphere, their configuration is restricted by the triangle inequality [6],

$$
3 V_{p}-\Gamma\left(\Gamma-2 \Gamma_{4}\right) b_{1} b_{2} b_{3} \geqslant 0,
$$

in which (a)

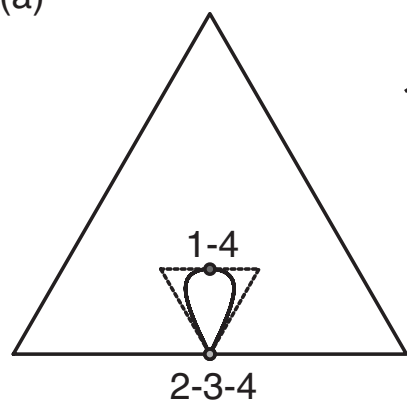

(b)

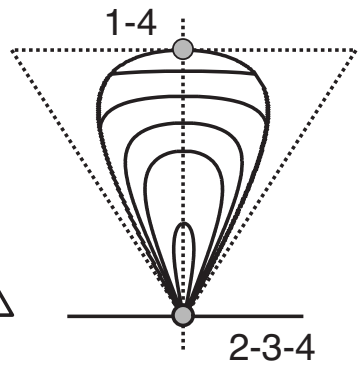

FIG. 3. (a) The trilinear triangle (the larger one), the physical triangle (the smaller one with dashed line), and the physical region (the solid closed curve inside the physical triangle) for $\Gamma_{1}=3, \Gamma_{2}$ $=2, \Gamma_{3}=2$, and $\Gamma=-1$. The point " $2-3-4$ " denotes the singular configuration where the vortex triple 234 is at the same position, and the point " $1-4$ " corresponds to the singular configuration where the first and the fourth point vortices are at the same position. (b) Contour lines of the Hamiltonian inside the physical region, which is a close-up of the left figure. The vertical dashed line in the center corresponds to the initial configuration of the four point vortices (18)

$$
\begin{aligned}
V_{p}= & 2 \Gamma_{2} \Gamma_{3} b_{2} b_{3}+2 \Gamma_{3} \Gamma_{1} b_{3} b_{1}+2 \Gamma_{1} \Gamma_{2} b_{1} b_{2} \\
& -\left(\Gamma_{1} b_{1}\right)^{2}-\left(\Gamma_{2} b_{2}\right)^{2}-\left(\Gamma_{3} b_{3}\right)^{2} .
\end{aligned}
$$

The region where the condition (16) is satisfied is referred to as the physical region. The configuration where the three point vortices lie on a great circle corresponds to a point at the boundary of the physical region. The solution of the integrable four-vortex problem evolves along a contour curve of Hamiltonian,

$$
\begin{aligned}
H= & \Gamma_{2} \Gamma_{3} \log \left|b_{1}\right|+\Gamma_{3} \Gamma_{1} \log \left|b_{2}\right|+\Gamma_{1} \Gamma_{2} \log \left|b_{3}\right| \\
& +\Gamma_{1} \Gamma_{4} \log \left|B_{1}\right|+\Gamma_{2} \Gamma_{4} \log \left|B_{2}\right|+\Gamma_{3} \Gamma_{4} \log \left|B_{3}\right| .
\end{aligned}
$$

Hence, we have only to plot contour lines of the Hamiltonian inside the physical region in the trilinear phase space to see the evolution of the four point vortices. The topological structure of contour lines of the Hamiltonian for the vortex strengths in the subregions $I$ to $I V$ has already been studied in [6]. On the other hand, less attention was paid to the motion of the four point vortices when the vortex strengths are chosen from the boundary lines between the subregions, where the triple 234 collapse is possible. We consider this case in the next section.

\section{NON-SELF-SIMILAR PARTIAL COLLAPSE}

We deal with a special case of $\Gamma_{1}=3, \Gamma_{2}=2, \Gamma_{3}=2$, and $\Gamma_{4}=-1$ that satisfies the conditions (8), (9), and (11), for which the collapse of the triple 234 is possible. In Fig. 3(a), the larger triangle represents the trilinear triangle and the smaller one is the physical triangle. Inside the physical triangle, the boundary of the physical region is drawn as a solid closed curve. Figure 3(b) is a close-up of the physical triangle, in which contour lines of the Hamiltonian are plotted. The two filled circles at the boundary of the physical region represent singular configurations corresponding to the triple 


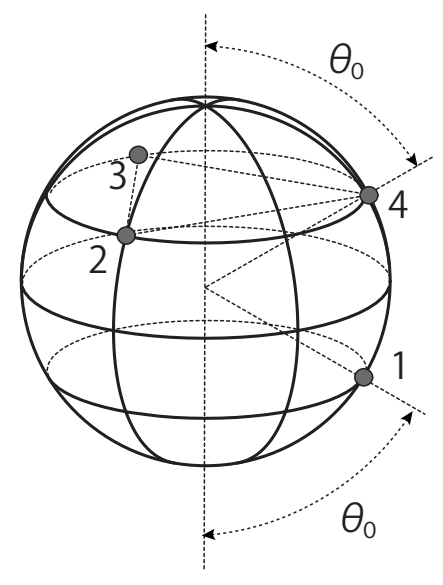

FIG. 4. Initial configuration for the four point voritces (18).

234 collapse, $b_{1}=B_{2}=B_{3}=0$, and the binary collapse of the vortices 1 and $4, B_{1}=0$, respectively. The contour lines appear to converge at the point of the triple 234 collapse, which suggests that the triple collapse occurs. We will discuss how the contour lines of the Hamiltonian behave in the neighborhood of the triple 234 collapse in the next section.

The above observation is verified by numerical means. Let $\Theta_{0} \neq 0$ be a parameter. Then we give an initial configuration of the four point vortices as follows:

$$
\begin{aligned}
& \theta_{1}=\pi-\Theta_{0}, \quad \theta_{2}=\theta_{3}=\theta_{4}=\Theta_{0}, \\
& \phi_{1}=\phi_{4}=0, \quad \phi_{2}=-\phi_{3}=2 / 3 \pi,
\end{aligned}
$$

in which the vortex triple 234 forms an equilateral triangle at the line of latitude $\Theta_{0}$ and the first point vortex is located at the position symmetric to the fourth point vortex with respect to the equator, as we see in Fig. 4. The trilinear coordinates for this configuration are given by

$$
b_{1}=\frac{3}{4} \sin ^{2} \Theta_{0}, \quad b_{2}=b_{3}=\frac{3}{8}\left(4-\sin ^{2} \Theta_{0}\right),
$$

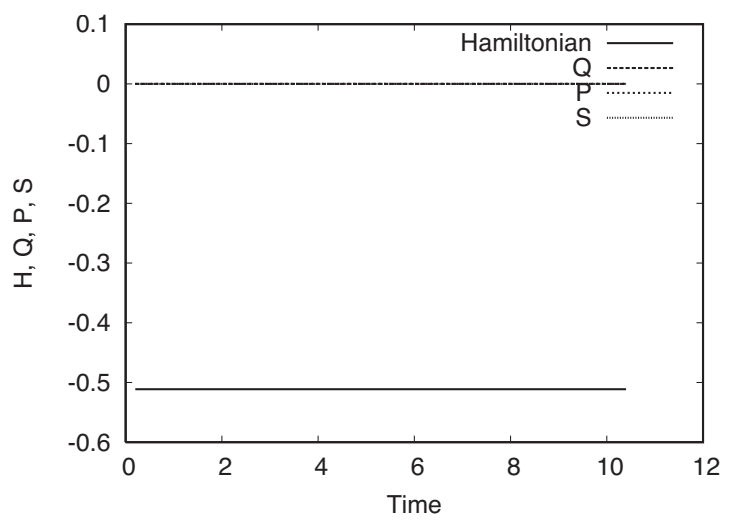

FIG. 5. Plot of the values of the Hamiltonian $H$ and the moment of vorticity vector $(Q, P, S)$ for the initial configuration (18) with $\Theta_{0}=0.2 \pi$. blackWhile the value of $H=-0.5113603555$ is unchanged up to 10 digits, those of $Q, P$, and $S$ stay zero within $O\left(10^{-10}\right)$ until the collapse time.

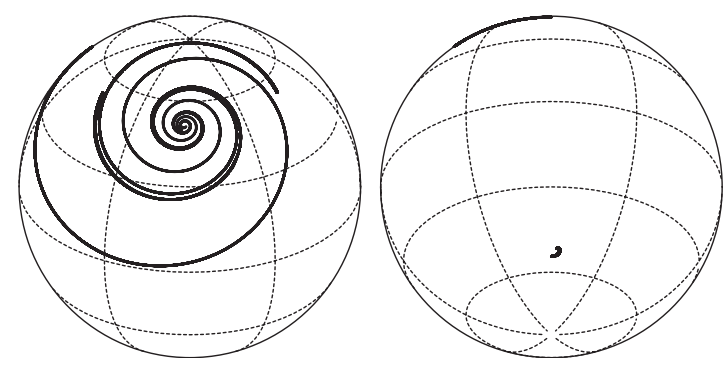

FIG. 6. Collapse of the vortex triple 234 for the initial configuration (18) with $\Theta_{0}=0.2 \pi$. The first point vortex moves toward the antipodal position to the collapse point when the triple collapse occurs.

$$
B_{1}=\frac{3}{4}\left(\sin ^{2} \Theta_{0}-1\right), \quad B_{2}=B_{3}=-\frac{3}{8} \sin ^{2} \Theta_{0},
$$

which means that the initial configuration (18) corresponds to the center line $b_{2}=b_{3}$ and $B_{2}=B_{3}$ in the trilinear and the physical phase spaces, which is drawn as a vertical dashed line in Fig. 3(b). It follows from the triangle inequality (16) and the trilinear coordinates (19) that the parameter $\Theta_{0}$ varies in the range of $(0, \pi / 2)$. For $\Theta_{0}=0$, the initial configuration corresponds to the collapse of vortex triple 234, while the first point vortex coincides with the fourth one at the equator for $\Theta_{0}=\pi / 2$.

Regarding the temporal integration of Eq. (2), we use the fourth-order Runge-Kutta method with time step size $\Delta t=10^{-5}$ and we stop the numerical computation when the relative distances between the vortex triple 234 become less than a threshold value $10^{-5}$. We verify the accuracy of the numerical computation by checking the values of the Hamiltonian and the moment of vorticity vector at each time step. In the examples of this paper, these values remain the same as those for the initial configuration up to six-digits at most. For example, we plot the values of the Hamiltonian and the moment of vorticity vector for the initial configuration (18) with $\Theta_{0}=0.2 \pi$ in Fig. 5. Although the vortex triple 234 collapses for this case as shown later, we hardly see any variation in these values.

We show the trajectory of the four point vortices for the initial data (18) with $\Theta_{0}=0.2 \pi$ in Fig. 6, which indicates that the vortex triple 234 collapses to one point at some finite time, say $t_{c}$, and the first point vortex stays away from the collapsing point. In order to see the trajectory quantitatively, we plot the relative distances $l_{m j}^{2}$ in Fig. 7. It shows that the distances between the vortex triple 234 tend to zero, while those between the first point vortex and the triple approach 4 , i.e., $l_{23}^{2}, l_{24}^{2}, l_{34}^{2} \rightarrow 0$ and $l_{12}^{2}, l_{13}^{2}, l_{14}^{2} \rightarrow 4$. Hence, the first vortex is located at the antipodal position to the collapsing point. Then, we pay attention to the ratios $l_{24}^{2} / l_{23}^{2}$ and $l_{34}^{2} / l_{23}^{2}$ between the vortex triple 234. If they are constant throughout the evolution, the collapse is self-similar. However, as we see in Fig. 8, the ratios change and tend to different constants asymptotically as time approaches the collapse time. This means the collapse of the vortex triple is non-self-similar.

Now we change the parameter $\Theta_{0}$. Figure 9 shows the distances between the four point vortices and the ratios of the 


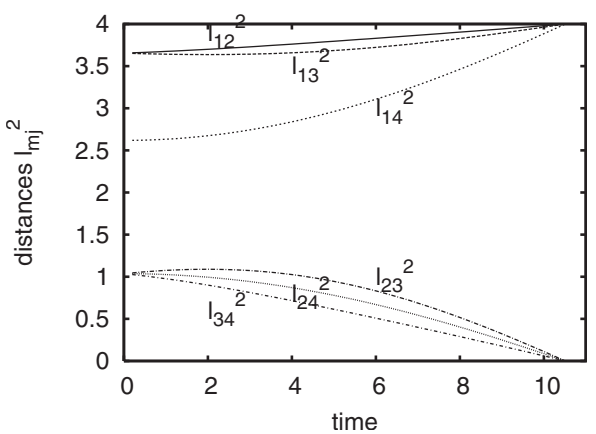

FIG. 7. Evolution of the relative distances $l_{m j}^{2}$ between the four point vortices for the collapsing orbit in Fig. 6.

vortex triple 234 for the initial configuration (18) with $\Theta_{0}$ $=0.3 \pi$. This indicates that non-self-similar triple collapse occurs for this case. We observe the same type of non-selfsimilar triple 234 collapse for $\Theta_{0} \in[0.04 \pi, 0.3553 \pi]$. Let us note that it is difficult to compute the triple collapse accurately for $\Theta_{0} \leqslant 0.04 \pi$ by numerical means, since the initial configuration is close to the singular configuration. Then we plot the collapse time $t_{c}$ for various $\Theta_{0}$ in Fig. 10. The collapse time increases rapidly as $\Theta_{0}$ tends to $0.3553 \pi$. This implies that there exists a threshold value $\Theta_{c}$, where the collapse time blows up. As a matter of fact, if we take the parameter $\Theta_{0}$ a little larger, e.g., $\Theta_{0}=0.3554 \pi$, the vortex triple never collides and the orbit becomes periodic as in Fig. 11. For $0.3554 \pi \leqslant \Theta_{0}<\pi / 2$, we confirm that the evolution of the four point vortices is always periodic.

From the viewpoint of the trilinear representation in Fig. 3(b), all contour lines passing across the vertical center line $b_{2}=b_{3}$ for $0<\Theta_{0}<\Theta_{c}$ represent the orbits that lead to the triple 234 collapse in finite time. Since we can take any point in these contour lines as an initial configuration of the four point vortices, the triple collapse is robust under any perturbation of the initial configuration as long as the moment of vorticity vector remains zero.

\section{COLLAPSE AND INTEGRABILITY}

In the preceding section, we have found the collapse solution in the integrable four-vortex problem for a special choice of the vortex strengths that satisfies the necessary conditions (8) and (9) for the self-similar triple 234 collapse,

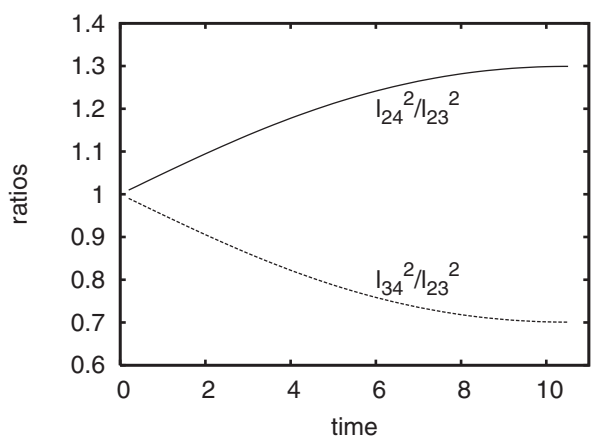

FIG. 8. Plots of $l_{24}^{2} / l_{23}^{2}$ and $l_{34}^{2} / l_{23}^{2}$, which are ratios of distances between the vortex triple 234, for the orbit in Fig. 6 .
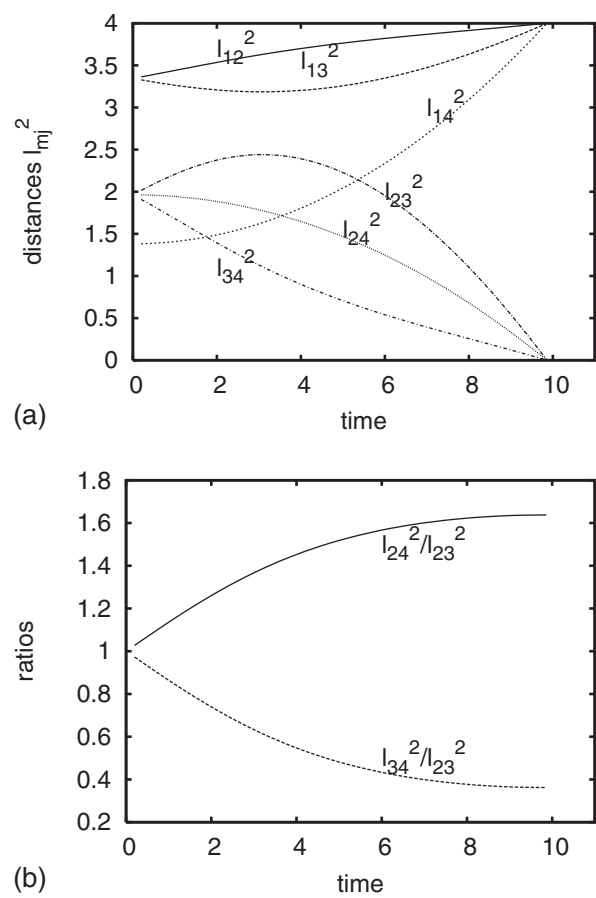

FIG. 9. Evolution of (a) the relative distances $l_{m j}^{2}$ between the four-point vortices, and (b) the ratios of the relative distances of the vortex triple 234 for the initial configuration (18) with $\Theta_{0}=0.3 \pi$.

which gives rise to some questions. The first one is whether we can observe non-self-similar collapse for other values of the vortex strengths. The second one is whether it is really necessary to assume the condition (9) for the vortex strengths. It is a necessary condition for the existence of self-similar collapse, but the triple 234 collapse we found is non-self-similar. The final one is how the integrability of the system plays an important role in the triple collapse. These are examined in this section with more examples.

First, we see the other integrable cases when the vortex strengths satisfy the necessary conditions (8) and (9) as in the preceding section. Here, we set the initial configuration as

$$
\theta_{2}=\theta_{3}=\theta_{4}=\Theta_{0}, \quad \phi_{2}=0, \quad \phi_{3}=2 \pi / 3 .
$$

The initial data for $\theta_{1}, \phi_{1}$, and $\phi_{4}$ are determined by solving $Q=P=S=0$ numerically. The parameter $\Theta_{0}$ is variable in the range of $(0,0.5 \pi)$. For Eq. (20) with $\Theta_{0}=0$, the vortex triple 234 is at the north pole and the first vortex is at the south

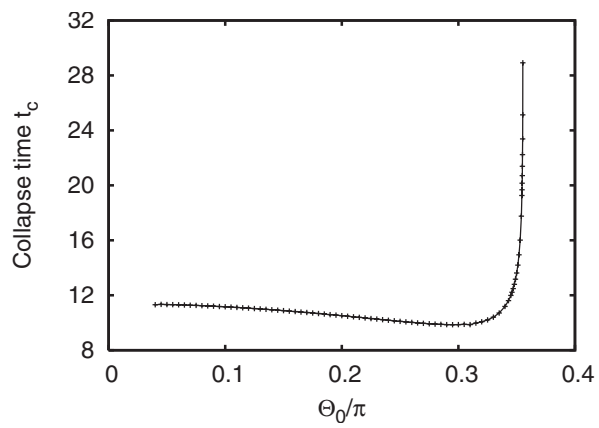

FIG. 10. Time of the triple 234 collapse, $t_{c}$, versus $\Theta_{0}$. 


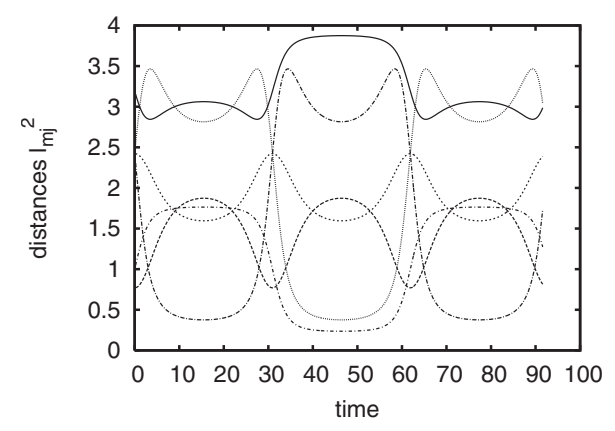

FIG. 11. Evolution of the relative distances $l_{m j}^{2}$ for the initial configuration (18) with $\Theta_{0}=0.3554 \pi$, which shows that the motion of the four vortices is periodic.

pole due to $S=0$ and Eq. (8). It corresponds to the singular triple 234 collapse. On the other hand, for $\Theta_{0}=0.5 \pi$, all the point vortices are in the equator and the first and the fourth point vortices are located at the same position, which corresponds to the singular binary 14 collapse. Figure 12 shows the evolution of the relative distances between the four-point vortices and the ratios between the vortex triple 234 for $\Gamma_{1}$ $=4, \Gamma_{2}=3, \Gamma_{3}=\frac{1}{2}(1+\sqrt{13})$, and $\Gamma_{4}=\frac{1}{2}(1-\sqrt{13})$, which indicates that the non-self-similar triple collapse also occurs. We plot contour lines of the Hamiltonian in the physical triangle in Fig. 13(a), and the collapse time $t_{c}$ of the triple 234 for the initial configuration (20) with $\Theta_{0} \in[0.05 \pi, 0.364 \pi]$ in Fig. 13(b). The collapse time $t_{c}$ blows up as $\Theta_{0}$ approaches $0.346 \pi$. The same phenomenon is also observed for the other case when $\Gamma_{1}=6, \Gamma_{2}=5, \Gamma_{3}=\frac{1}{2}(1+\sqrt{21})$, and $\Gamma_{4}=\frac{1}{2}(1-\sqrt{21})$, for which contour lines of the Hamiltonian in the physical triangle and the collapse time $t_{c}$ for the initial configuration
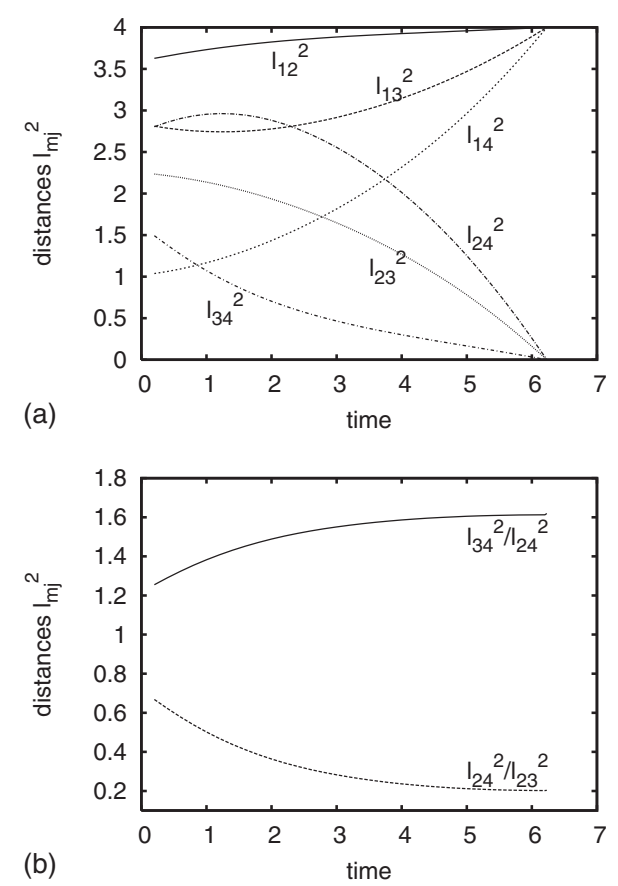

FIG. 12. Evolution of (a) the relative distances $l_{m j}^{2}$ between the four point vortices and (b) the ratios of the relative distances between the vortex triple 234 when $\Gamma_{1}=4, \Gamma_{2}=3, \Gamma_{3}=\frac{1}{2}(1+\sqrt{13})$, and $\Gamma_{4}=\frac{1}{2}(1-\sqrt{13})$. (a)
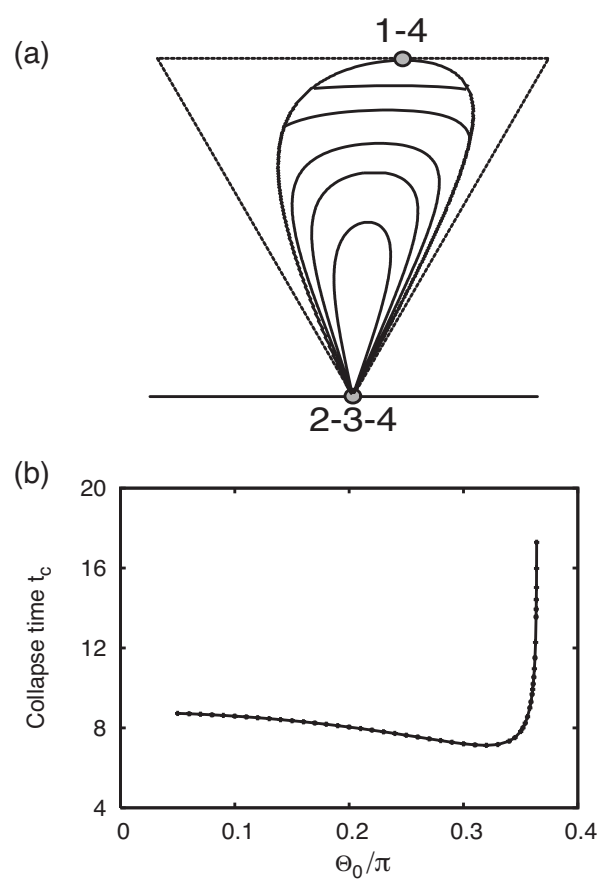

FIG. 13. (a) Contour lines of the Hamiltonian and (b) collapse time $t_{c}$ for $\Theta_{0} \in[0.05 \pi, 0.346 \pi]$ when $\Gamma_{1}=4, \quad \Gamma_{2}=3, \quad \Gamma_{3}=\frac{1}{2}(1$ $+\sqrt{13})$, and $\Gamma_{4}=\frac{1}{2}(1-\sqrt{13})$.

(20) with $\Theta_{0} \in[0.05 \pi, 0.3776 \pi]$ are shown in Figs. 14(a) and $14(\mathrm{~b})$, respectively.

Next, we consider the case in which $\Gamma_{1}=3, \Gamma_{2}=2, \Gamma_{3}$ $=1.5$, and $\Gamma_{4}=-0.5$ that satisfies Eq. (8) but not Eq. (9). Figure 15 shows the contour plot of the Hamiltonian in the
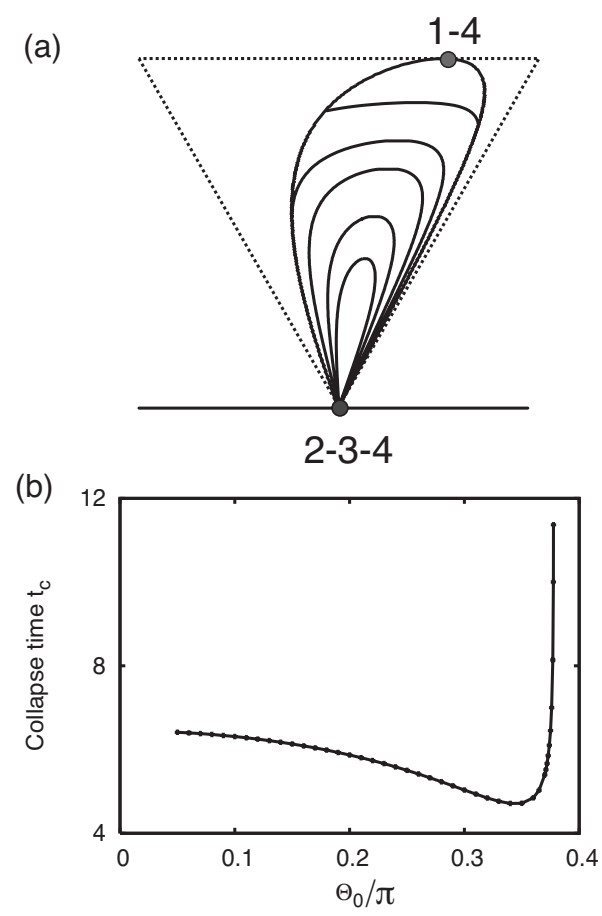

FIG. 14. (a) Contour lines of the Hamiltonian and (b) collapse time $t_{c}$ for $\Theta_{0} \in[0.05 \pi, 0.3776 \pi]$ when $\Gamma_{1}=6, \Gamma_{2}=5, \Gamma_{3}=\frac{1}{2}(1$ $+\sqrt{21})$, and $\Gamma_{4}=\frac{1}{2}(1-\sqrt{21})$. 


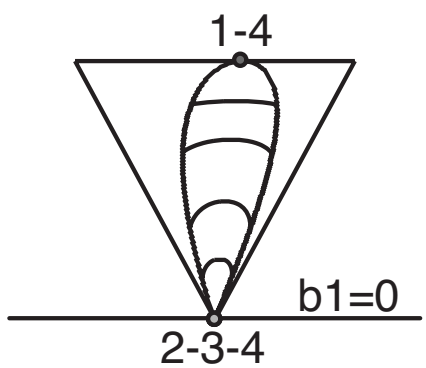

FIG. 15. Contour lines of the Hamiltonian for $\Gamma_{1}=3, \Gamma_{2}=2$, $\Gamma_{3}=1.5$, and $\Gamma_{4}=-0.5$ that satisfies Eq. (8) but not Eq. (9).

physical triangle, which shows that the contour lines never connect to the point corresponding to the triple 234 collapse. To confirm this observation, we consider the following initial configuration:

$$
\theta_{2}=\theta_{3}=\theta_{4}=\Theta_{0}, \quad \phi_{2}=\pi / 4, \quad \phi_{3}=3 / 4 \pi .
$$

The other variables, $\theta_{1}, \phi_{1}$ and $\phi_{4}$, are numerically determined by solving the equations $Q=P=S=0$. Note again that the initial configurations $(21)$ with $\Theta_{0}=0$ and $0.5 \pi$ correspond to the triple 234 collapse and the binary 14 collapse, respectively. As we change the parameter $\Theta_{0}$, the point corresponding to the initial configuration (21) in the trilinear phase space moves along a continuous curve that connects the two singular points and is transverse to contour lines of the Hamiltonian in Fig. 15. We numerically confirm that all orbits starting from the configuration (21) become periodic. Figure 16 shows the period for these periodic orbits for $\Theta_{0}$ $\in[0.05 \pi, 0.46 \pi]$. Hence, the necessary condition (9) for the self-similar triple 234 collapse is required for the existence of non-self-similar collapse.

We discuss here why the condition (9) is required for the existence of triple collapse. As we have observed in the three examples that satisfy Eq. (9), the contour lines of the Hamiltonian shown in Figs. 3, 13(a), and 14(a) appear to converge to the point corresponding to the triple 234 collapse. So we see how the contour lines behave in the neighborhood of the collapse point. It follows from Eqs. (8), (9), (14), and (15) that

$$
B_{2}=b_{2}-\frac{3 \Gamma_{3}}{\Gamma_{2}+\Gamma_{3}}, \quad B_{3}=b_{3}-\frac{3 \Gamma_{2}}{\Gamma_{2}+\Gamma_{3}} .
$$

Hence, the triple 234 collapse, i.e., $b_{1}=B_{2}=B_{3}=0$, is represented by

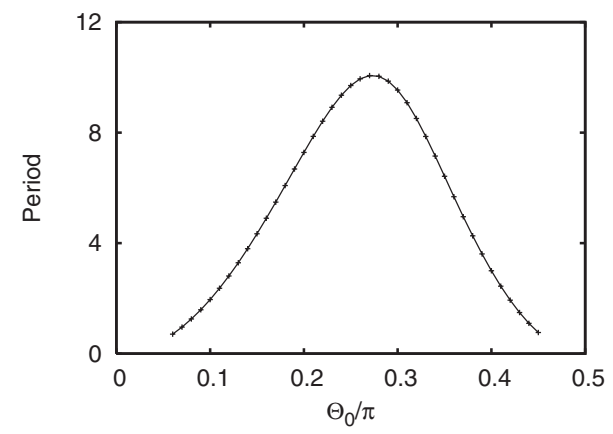

FIG. 16. Period of the periodic orbits for the initial configuration (21) versus $\Theta_{0}$ for $\Gamma_{1}=3, \Gamma_{2}=2, \Gamma_{3}=1.5$, and $\Gamma_{4}=-0.5$.

$$
\left(b_{1}, b_{2}, b_{3}\right)=\left(0, \frac{3 \Gamma_{3}}{\Gamma_{2}+\Gamma_{3}}, \frac{3 \Gamma_{2}}{\Gamma_{2}+\Gamma_{3}}\right)
$$

in the trilinear coordinates. Thus the trilinear coordinates of points near Eq. (22) are given by

$$
\begin{gathered}
b_{1}=s, \\
b_{2}=\frac{3 \Gamma_{3}}{\Gamma_{2}+\Gamma_{3}}-\frac{1}{2} s-\frac{\sqrt{3}}{2} \alpha, \\
b_{3}=\frac{3 \Gamma_{2}}{\Gamma_{2}+\Gamma_{3}}-\frac{1}{2} s+\frac{\sqrt{3}}{2} \alpha,
\end{gathered}
$$

in which $s$ and $\alpha$ are two independent parameters. Then the triple $\left(B_{1}, B_{2}, B_{3}\right)$ is represented by

$$
\begin{aligned}
& B_{1}=s+\frac{3 \Gamma_{4}}{\Gamma_{2}+\Gamma_{3}}, \\
& B_{2}=-\frac{1}{2} s-\frac{\sqrt{3}}{2} \alpha, \\
& B_{3}=-\frac{1}{2} s+\frac{\sqrt{3}}{2} \alpha .
\end{aligned}
$$

Due to Eq. (9), the Hamiltonian Eq. (17) becomes

$$
\begin{aligned}
H= & \Gamma_{2} \Gamma_{4} \log \left|\frac{B_{2}}{b_{1}}\right|+\Gamma_{3} \Gamma_{4} \log \left|\frac{B_{3}}{b_{1}}\right|+\Gamma_{1} \Gamma_{3} \log \left|b_{2}\right| \\
& +\Gamma_{1} \Gamma_{2} \log \left|b_{3}\right|+\Gamma_{1} \Gamma_{4} \log \left|B_{1}\right|,
\end{aligned}
$$

in which the first two terms become singular and the last three terms are finite as $s, \alpha \rightarrow 0$. However, as a matter of fact, owing to Eqs. (23), (27), and (28), the singular part behaves like

$$
\Gamma_{2} \Gamma_{4} \log \left|\frac{1}{2}+\frac{\sqrt{3}}{2} \frac{\alpha}{s}\right|+\Gamma_{3} \Gamma_{4} \log \left|\frac{1}{2}-\frac{\sqrt{3}}{2} \frac{\alpha}{s}\right|,
$$

which is finite when we take the limit $s, \alpha \rightarrow 0$ with the ratio $\alpha / s$ being constant. Moreover, the limit value of the singular part depends on the ratio $\alpha / s$, which allows the contour lines of the Hamiltonian to converge at the triple 234 collapse. Consequently, the condition (9) is necessary for the existence of the triple 234 collapse.

Finally, we perturb the initial configuration (18) so that it loses its integrability. The initial positions of the second, the third, and the fourth point vortices are the same as in Eq. (18), but the first point vortex is slightly perturbed as $\theta_{1}$ $=\pi-\Theta_{0}+\varepsilon$, in which $\varepsilon=10^{-4}$ and $\Theta_{0}=0.2 \pi$. Then the fourvortex problem is no longer integrable since $Q \neq 0$ and $S$ $\neq 0$ at the initial moment. Figure 17 shows the trajectory of the four point vortices. After the vortex triple 234 approaches closely, the three point vortices swing by and separate away. The evolution of the relative distances $l_{m j}^{2}$ between the fourpoint vortices is plotted in Fig. 18(a). It seems that no collapse of the four point vortices occurs. We confirm this by plotting the evolution of the vortex triple 234 around $t$ 


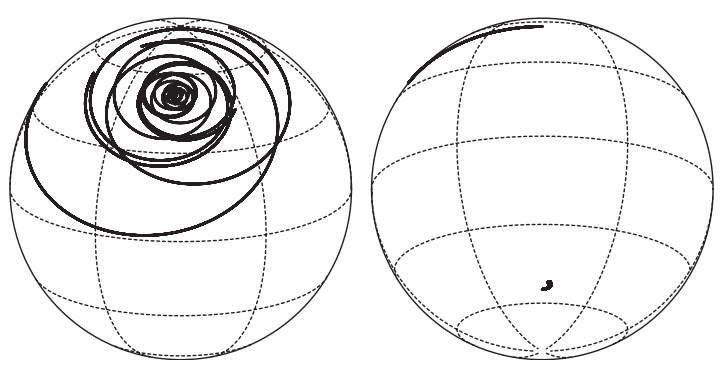

FIG. 17. Trajectory of the four-point vortices when the initial configuration (18) is perturbed as $\theta_{1}=\Theta_{0}+\varepsilon$ with $\varepsilon=10^{-4}$ and $\Theta_{0}$ $=0.2 \pi$. The strengths of the point vortices are $\Gamma_{1}=3, \Gamma_{2}=2, \Gamma_{3}=2$, and $\Gamma_{4}=-1$. The four-vortex problem on the sphere is not integrable for this initial configuration.

$\approx 10.5$ with a $\log$ plot on the vertical axis in Fig. 18(b), which shows that the relative distances are greater than $10^{-5}$. This example indicates that the integrability of the system is also necessary for the existence of non-self-similar collapse.

\section{SUMMARY AND DISCUSSION}

We have reported some numerical examples showing that non-self-similar triple collapse is possible in the integrable four-vortex problem on the sphere. Three point vortices collide at one point in finite time and the other one is located at the antipodal position to the collision point. The non-selfsimilar triple collapse has never been reported so far and it is a unique phenomenon found in the four-vortex problem on the sphere. It would be interesting to see if the partial collapse occurs in the planar four-vortex problem or not.

The singular solution is robust under the perturbation of initial data as long as the Hamiltonian system remains integrable. This is different from the self-similar collapse in the three-vortex problem found in the preceding research, since the choice of the initial configuration for the collapse is strongly restricted, i.e., it is nongeneric. We also see that the integrability of the system is a necessary condition for the existence of non-self-similar triple collapse.

Let us finally note that the present paper does not give a rigorous mathematical proof for the existence of non-selfsimilar collapse. Although we have checked the numerical results as carefully as possible, we are unable to avoid a certain approximation error and the round-off error in nu-
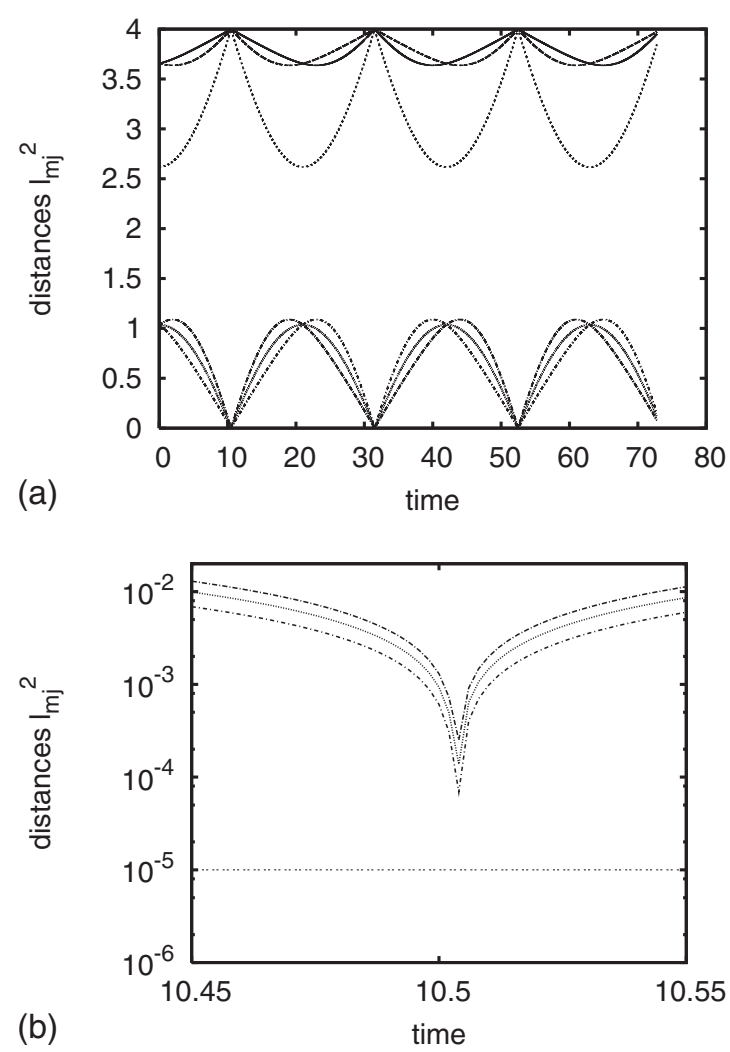

FIG. 18. (a) Evolution of the relative distances $l_{m j}^{2}$ between the four point vortices corresponding to the trajectory in Fig. 17. (b) Log-plot of the evolution of the vortex triple 234 around $t \approx 10.5$. The dotted line represents the value of $10^{-5}$.

merical computations. Nevertheless, the examples still give us some useful information for the mathematical proof. For instance, the conditions on the vortex strengths (8) and (9) and the integrability of the Hamiltonian system are necessary for the existence of non-self-similar, partial, and robust collapse. We have tried to describe the singular solution explicitly, but we have been unsuccessful as of yet. A topological regularization method proposed by Hiraoka [11] for the planar three-vortex problem could be applied to prove the existence of non-self-similar collapse, but its application to the four-vortex problem on the sphere is not straightforward. We need more research on the topic, and it will be reported on in the near future.
[1] P. K. Newton, The N-Vortex Problem, Analytical Techniques (Springer-Verlag, New York, 2001).

[2] H. Aref, Phys. Fluids 22, 393 (1979).

[3] R. Kidambi and P. K. Newton, Physica D 116, 95 (1998).

[4] T. Sakajo, Jpn. J. Ind. Appl. Math. 16, 321 (1999).

[5] S. L. Ziglin, Sov. Math. Dokl. 21, 296 (1980).

[6] T. Sakajo, Phys. Fluids 19, 017109 (2007).
[7] H. Aref and M. S. Stremler, Phys. Fluids 11, 3704 (1999).

[8] B. Eckhardt, Phys. Fluids 31, 2796 (1988).

[9] Y. Kimura, Fluid Dyn. Res. 3, 98 (1988).

[10] R. Kidambi and P. K. Newton, Nuovo Cimento Soc. Ital. Fis., C 22C, 779 (1999).

[11] Y. Hiraoka, Nonlinearity 21, 361 (2007).

[12] N. Rott, Phys. Fluids A 2, 1477 (1990). 